\title{
rno-miR-128-3p promotes apoptosis in rat granulosa cells (GCs) induced by norepinephrine through Wilms tumor 1 (WT1)
}

\author{
Ming $\mathrm{Li}^{1} \cdot$ Ling Xue ${ }^{2} \cdot$ Weibin $\mathrm{Xu}^{3} \cdot$ Pingping $\mathrm{Liu}^{4} \cdot$ Feng $\mathrm{Li}^{5}$
}

Received: 11 April 2021 / Accepted: 7 July 2021 / Published online: 23 September 2021 / Editor: Tetsuji Okamoto

(C) The Author(s) 2021

\begin{abstract}
The mechanism related to ovarian follicular is complex, which has not been fully elucidated. Abundant reports have confirmed that the ovarian function development is closely related to sympathetic innervation. As one of the major neurotransmitters, norepinephrine (NE) is considered an effective regulator of ovarian functions like granulosa cell (GC) apoptosis. However, the mechanism between NE and GC apoptosis in rat is still unclear. In our study, GCs were isolated and cultured in vitro with NE treatment. The apoptosis of GCs was facilitated by NE. Wilms tumor 1 (WT1) was found to be significantly downregulated in GCs after NE treatment, and overexpression of WT1 repressed apoptosis in rat GCs induced by NE. rno-miR-128-3p was found to be significantly enhanced by NE in GCs, and inhibition of rno-miR-128-3p repressed apoptosis in rat GCs induced by NE. Mechanistically, rno-miR-128-3p interacted with WT1 and repressed its expression. In summary, inhibition of rnomiR-128-3p may enhance WT1 expression, and then repress NE-induced apoptosis in rat GCs. Our research may provide a new insight for the improvement of ovarian follicular development.
\end{abstract}

Keywords Norepinephrine $\cdot$ Rno-miR-128-3p $\cdot$ WT1 $\cdot$ Granulosa cell

\section{Introduction}

Follicle development is a complex physiological process that is crucial to ovarian function and fertility (Fortune 1994). When the follicles begin to develop, the static follicles are

Feng Li

lf19936568728@163.com

1 Department of Pharmacy, Lian Yungang Hospital of Traditional Chinese Medicine, Affiliated Hospital of Nanjing University of Chinese Medicine, Jiang Su Province, Lianyungang 222000, People's Republic of China

2 Pharmacy Department, Shandong Qingdao Hospital of Integrated Traditional Chinese and Western Medicine, No. 3 Jiaxiang Road, South District, Qingdao City, Shandong Province 266002, China

3 Pharmacy Department, Gaoqing County People's Hospital, No.11 Qingcheng Road, Gaoqing County, Zibo City 256300, Shandong Province, China

4 Pharmacy Department, Liaocheng Chiping District People's Hospital, No. 1057 Culture Road, Chiping County, Liaocheng City 252100, Shandong Province, China

5 Drug Dispensing Department, Zibo Central Hospital, No. 54 The Communist Youth League West Road, Zhangdian District, Zibo City 255000, Shandong Province, China activated and gradually result in the growth and selection of dominant follicles (Murray and Spears 2000; Webb 2003). Interruption of follicle development may lead to an increase in follicular atresia, which is a main reason for ovarian pathology (Voorhis 2008). Hence, investigating the potential mechanisms of follicle development is crucial for the treatment of ovarian disease.

As one of the major neurotransmitters, norepinephrine (NE) is considered an effective regulator of ovarian functions like granulosa cell (GC) apoptosis (Zhang et al. 2017a). There are two pathways to the sympathetic innervation in the rodent ovaries, including the upper nerves of the ovary extending along the ovary ligaments and the plexus nerves mainly related to the vasculature (Campo 2019). NE level plays a crucial role in regulating ovarian cystic health. In ovarian cancer, NE stimulated cell invasion by elevating hTERT expression (Choi 2015). A study conducted by Patel et al. (2015) found that NE decreases ROS and DNA injury in ovarian surface epithelial cells. NE is reported to be related with the ROS production in GCs, which acts as important factors in ovarian physiology, like ovulation (Saller 2012). However, it is not clear whether and how NE affects the GC apoptosis in rat. 
MicroRNA ( $\mathrm{miR} / \mathrm{miRNA}$ ) is a small RNA that can directly bind to the 3'UTR of targeted mRNA, as a result of mRNA degradation. miRNA represses gene transcription of target (Hu 2018). Vast evidences revealed that miRNAs played pivotal roles in ovarian disease, like polycystic ovary syndrome (PCOS) (Huang 2020) and ovarian cancer (Feng et al. 2020). A study conducted by Wang et al. (2020) found that miR-29 regulates GC function through suppressing PTX3. And miR-130b is involved in the development of PCOS through regulating Cx43 (Jiang et al. 2020). miR$128-3 p$ is reported to be elevated in GCs of diminished ovarian reserve (Woo 2018), but whether and how miR-128-3p affects the GC apoptosis was unknown.

The Wilms tumor gene 1 (WT1) encodes a zinc finger transcription factor in gonads, including testis and ovary (Pelletier 1991; Chun 1999). WT1 played crucial effects on the gonad development. In the ovaries, WT1 inhibition results in cell ectopic development, which in turn decreases ovary weight and the number of large sinus-shaped follicles (Gao 2014; Chen 2017). Besides, WT1 alters GC apoptosis and proliferation by regulating the $w n t / \beta$-catenin pathway (Wang et al. 2019). The expression of WT1 is regulated by miRNAs. For example, miR-361-5p represses the development of hepatocellular carcinoma by repressing WT1 (Cheng 2019). Magnesium isoglycyrrhizinate diminishes fructose-induced apoptosis of podocytes via decrease of miR-193a to upregulate WT1 (Li 2019a). The bioinformatics analysis predicted that miR-128-3p was a candidate to upregulate WT1. However, there was no relevant report on miR-128-3p and WT1. So we focus on microRNA and WT1 in the mechanism of norepinephrine-inducing apoptosis.

In this research, we aimed to investigate the effects of miR-128-3p on rat GCs. This study used GCs isolated from rat ovary as a cell model to evaluate the function of miR$128-3 p$ on GCs, and the underlying mechanism was illuminated by evaluating the relation between miR-128-3p and WT1. Our findings may offer a new insight for the improvement of ovarian follicular development.

\section{Materials and methods}

Ethical approval Sprague-Dawley (SD) rats (age $21 \mathrm{~d}$; weighing 45-55 g) were acquired from Shanghai Slyke Experimental Animals Co., Ltd. (Shanghai, China). Rats were treated humanely using approved procedures granted by the local Animal Ethics Committee.

GC culture and treatment Ovaries were collected and put in serum-free high-glucose Dulbecco's Modified Eagle's Medium (DMEM), followed by washing and suspending in DMEM with 5\% fetal bovine serum (FBS). After puncturing the follicle with the fine needles, the GCs are strongly precipitated and washed by using the medium. GCs were collected and cultured in DMEM with 10\% FBS (Shen and Wang 2019). The primary GCs were transfected after 48-h culture.

For NE treatment, NE is dissolved in DMSO; GCs were treated with $0,0.1,1$, and $10 \mu \mathrm{mol} / \mathrm{L} \mathrm{NE}$ for $24 \mathrm{~h}$, or treated with $10 \mu \mathrm{mol} / \mathrm{L} \mathrm{NE}$ for $0,6,12,24$, and $48 \mathrm{~h}$, respectively. The control experiment is all added with the DMSO vehicle for transfection; GCs were transfected for $6 \mathrm{~h}$ before NE treatment (Zhang et al. 2017b). Lipofectamine ${ }^{\text {TM }} 3000$ (Invitrogen, Thermo Fisher, Carlsbad, CA) was utilized. PcDNA-WT1, rno-miR-128-3p mimics, rno-miR-128-3p inhibitor, si-WT1, and negative control (NC) were obtained from Genepharma (Shanghai, China).

TUNEL assay Twenty-four hours after NE treatment, GCs were fixed using $2 \%$ formalin at $37^{\circ} \mathrm{C}$ for $1 \mathrm{~h}$, using $0.1 \%$ Triton X-100 to permeate on ice for $2 \mathrm{~min}$, followed by 1-h incubation with $50 \mu \mathrm{L}$ TUNEL reaction mixture at $37^{\circ} \mathrm{C}$ under dark conditions. The results were observed under fluorescence microscope (LSM880, Zeiss, Oberkochen, Germany) (Li 2019b).

Flow cytometry assay $1.00 \times 10^{6}$ cells $/ \mathrm{mL}$ was applied for PI staining for $10 \mathrm{~min}$ and subjected to cell apoptosis analysis using FITC and FACSCalibur flow cytometer $(342,975$, BD, Franklin Lakes, NJ). The number of cells was acquired at 488-nm wavelength and analyzed by Cell Quest software.

\section{RT-qPCR assay}

Total RNA in GCs was obtained by TRIzol (SigmaAldrich, St. Louis, St. Louis, MO) following the instructions. RT-PCR was carried by employing TaqMan ${ }^{\mathrm{TM}}$ MicroRNA Reverse Transcription Kit (Applied Biosystems ${ }^{\mathrm{TM}}$, Foster City, CA) for rno-miR-128-3p and RevertAid RT Reverse Transcription Kit (Thermo Scientific ${ }^{\mathrm{TM}}$, CA) for genes; qRT-PCR was performed as previously described (Wang 2019). The primers (Zhang et al. 2017b; Li 2019c; Zhang 2019, 2020; Harishkumar and Selvaraj 2020; Wu 2020) are listed in Table 1. U6 and GAPDH served as an endogenous control.

Western blot Cell lysate was isolated utilizing cell lysis buffer (Beyotime, Nanjing, China). Western blots were conducted accordingly as previously described (Chowdhury 2013). Briefly, the membrane was transferred and blocked at $37^{\circ} \mathrm{C}$ for $2 \mathrm{~h}$, followed by primary antibody incubation: rabbit anti-caspase-3 (1:500, ab13847), anti-cleaved caspase-3 (1:5000, ab49822), anti-caspase-9 (1:1000; ab184786), anticleaved caspase-9 (1:2000, ab2324), anti-BAX (1:5000; ab32503), anti-BCL2 (1:1000, ab59348), anti-WT1 (1:100, 
Table 1 Primer sequences for qRT-PCR

\begin{tabular}{ll}
\hline Primer name & $\left(\right.$ 5'-3') primer sequences $^{\prime}$ \\
\hline Caspase-3-forward & 5'-ATGTCGATGCAGCTAACCTC-3' \\
Caspase-3-reverse & 5'-TCCTTTTGCTGTGATCTTCC-3' \\
Caspase-9-forward & 5'-CTGAGCCAGATGCTGTCCCATA-3' \\
Caspase-9-reverse & 5'-GACACCATCCAAGGTCTCGAT \\
& GTA-3' \\
BAX-forward & 5'-TTTCATCCAGGATCGAGCAGG-3' \\
BAX-reverse & 5'-GCAAAGTAGAAGAGGGCA \\
& ACCAC-3' \\
BCL2-forward & 5'-CTACCGTCGTGACTTCGCA-3' \\
BCL2-reverse & 5'-TACCCAGCCTCCGTTATCC-3' \\
WT1-forward & 5'-GTGGCTCCTGCGTTTCCCCC-3' \\
WT1-reverse & 5'-CCACAGGCATG GCGCGG-3' \\
GAPDH-forward & 5'-ATGGCAGACGATGATCCCTAC-3' \\
GAPDH-reverse & 5'-CGGAATCGAAATCCCCTCTGTT-3' \\
rno-miR-128-3p-forward & 5'-GGTCACAGTGAACCGGTC-3' \\
rno-miR-128-3p-reverse & 5'-GTGCAGGGTCCGAGGT-3' \\
U6-forward & 5'-CTCGCTTCGGCAGCACA-3' \\
U6-reverse & 5'-AACGCTTCACGAATTTGCGT-3' \\
\hline
\end{tabular}

ab224806), and anti- $\beta$-actin (1:5000, ab179467), and then re-probed with $\operatorname{IgG}$ complexed to horseradish peroxidase (1:2000, ab6721) antibody. The chemiluminescence kit (Millipore, Darmstadt, Germany) was adopted to observe the immune response zone, and ImageJ software (ImageJ Software Inc., MD) was used to quantify the integrated density of each band. Antibodies mentioned before were supplied by Abcam (Cambridge, UK).

Dual luciferase reporter assay In order to confirm that WT1 was a target of rno-miR-128-3p, WT1-WT (containing the binding sites of rno-miR-128-3p at WT1 3'UTR) and WT1MUT (mutation of binding sites) were cloned into Luciferase Reporter Vector (AM5795, Invitrogen, Carlsbad,, CA). WT1-WT or WT1-MUT were co-transfected with rno-miR128-3p mimics into GCs. Forty-eight hours after transfection, GCs were explored using a Reporter Vector System (AM5795, Invitrogen, CA) via a Glomax20/20 luminometer (Promega, Madison, WI) (Zhang et al. 2017b).

Statistical analysis The mean \pm SD represented data from three independent experiments. SPSS 21.0 (IBM Corp., Armonk, NY) was applied for statistical analysis of all data. Student's $t$ test was used for comparison between two groups, and one-way ANOVA and Tukey's posttests were used for multiple groups. The level of significance was $P<0.05$.

\section{Results}

NE induces apoptosis in rat GCs and represses the expression levels of WT1 After treating with different concentrations of NE, the apoptosis of GCs was assessed by the TUNEL assay and flow cytometry assay. As shown in Fig. $1 A$ and $B$, the apoptosis was elevated in a NE concentration-dependent manner. RT-qPCR was conducted to assess the levels of caspase-3, caspase-9, BAX, and BCL2, and our data exhibited that the mRNA of caspase-3, caspase-9, and BAX was significantly improved by $\mathrm{NE}$ in a concentration-dependent manner, while the results of BCL2 were the opposite (Fig. 1C). What's more, the same phenomenon was observed via the Western blot assay, which exhibited that the protein of cleaved caspase-3, cleaved caspase-9, and BAX was significantly improved by NE in a concentration-dependent manner, while the results of BCL2 were the opposite (Fig. 1D).

Next, we further verified the effect of NE on the level of WT1. After being treated with different concentrations of NE, RT-qPCR and Western blot assay were conducted to assess the level of WT1. Our data exhibited that both mRNA and protein of WT1 were decreased by NE treatment and showed NE concentration dependence (Fig. 1E); then, $10 \mu \mathrm{mol} / \mathrm{L}$ NE was selected for subsequent experiments. Furthermore, GCs were treated with $10 \mu \mathrm{mol} / \mathrm{L}$ NE for 0 , $6,12,24$, and $48 \mathrm{~h}$, respectively. The results of RT-qPCR and Western blot assay exhibited that the level of WT1 was lowest for the time of $24 \mathrm{~h}$. As a result, 24-h treatment was selected for subsequent experiments.

Overexpression of WT1 represses apoptosis in NE-treated rat GCs After transfecting NE-treated GCs with pcDNA-WT1 to upregulate WT1 expression, the TUNEL assay and flow cytometry assay were conducted to assess the apoptosis of GCs. The data revealed that NE elevated GC apoptosis compared with the pcDNA-NC group, which was alleviated by pcDNA-WT1 (Fig. 2A and $B$ ). RT-qPCR was conducted to assess the levels of caspase-3, caspase-9, BAX, and BCL2, and our data exhibited that the mRNA of caspase-3, caspase-9, and BAX was significantly improved after being treated with NE, whereas it was reversed by pcDNA-WT1 and the results of BCL2 were the opposite (Fig. 2C). What's more, similar results were observed via the Western blot assay, which exhibited that the protein of cleaved caspase-3, cleaved caspase- 9 , and BAX was significantly improved by NE, whereas it was reversed by pcDNA-WT1 and the results of BCL2 were the opposite (Fig. $2 D$ ). Taken together, GC apoptosis induced by NE was repressed due to WT1 overexpression. 


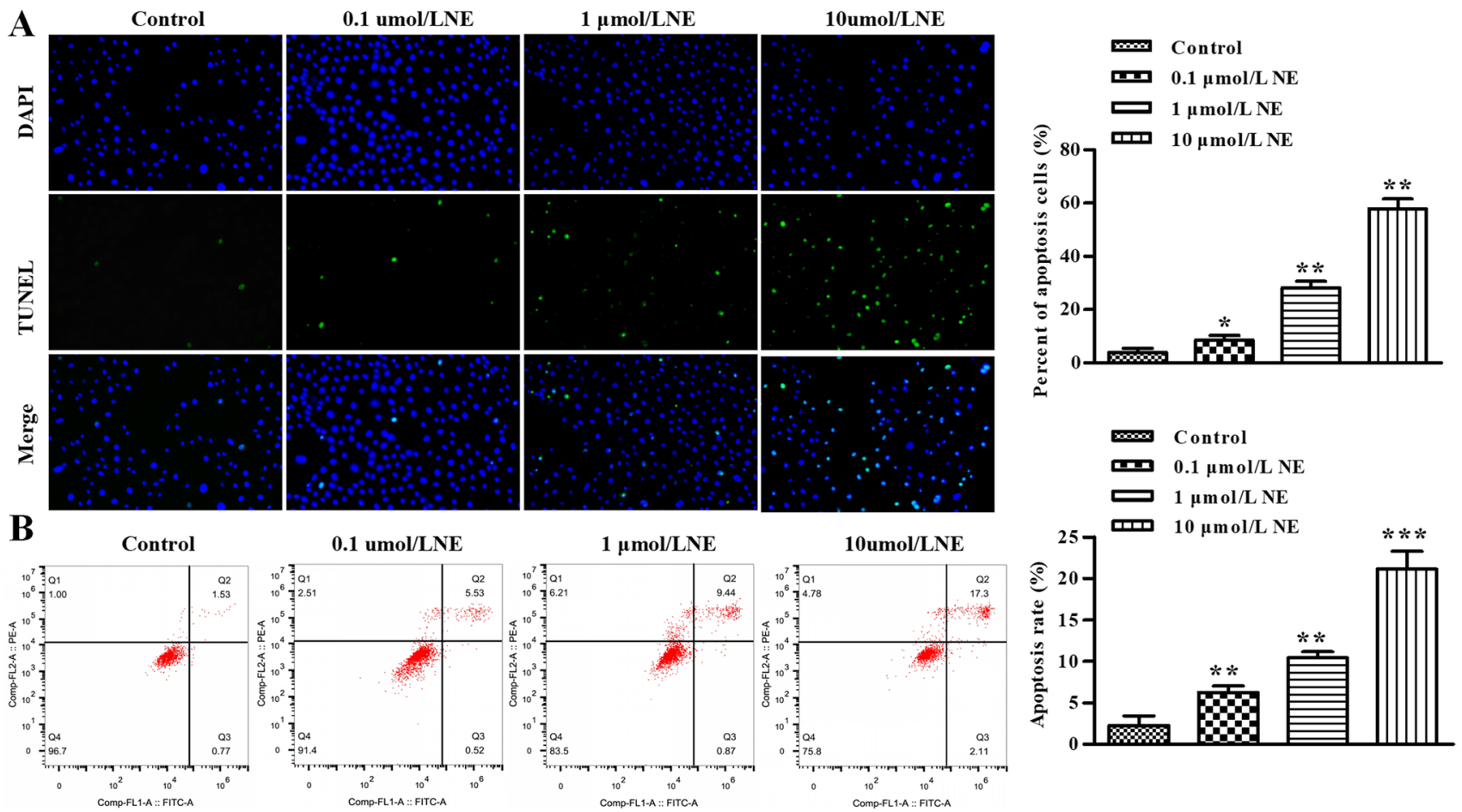

C

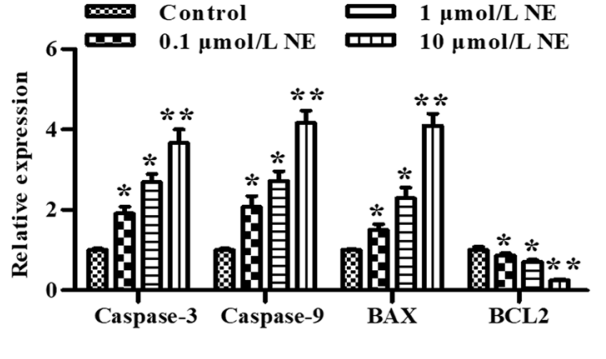

D

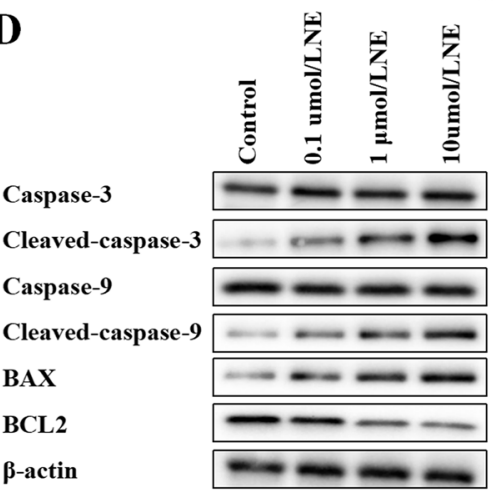

Control $1 \mu \mathrm{mol} / \mathrm{L} \mathrm{NE}$ שיח $0.1 \mu \mathrm{mol} / \mathrm{L} \mathrm{NE} 10 \mu \mathrm{mol} / \mathrm{L} \mathrm{NE}$

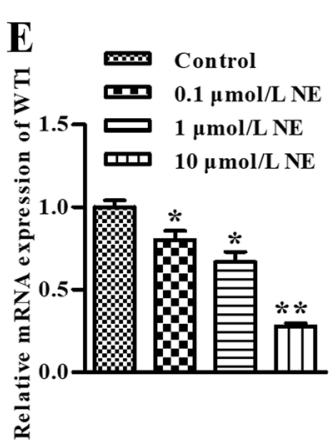

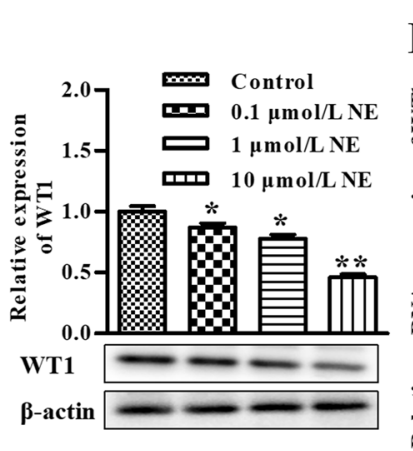

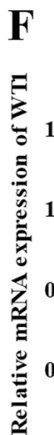

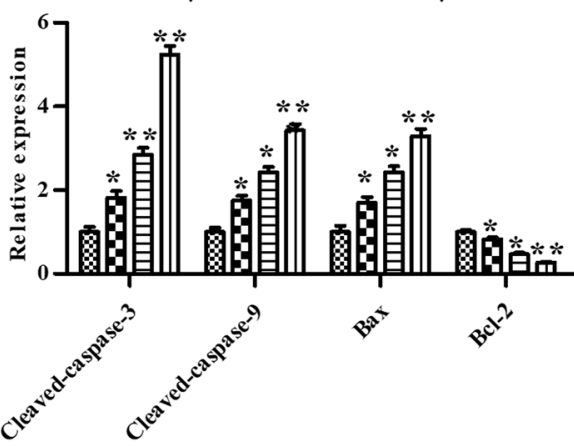

Figure 1. NE induces apoptosis in rat GCs and represses the expression levels of WT1. (A) The cell apoptosis was determined using TUNEL assay in rat GCs. (B) The cell apoptosis was determined using flow cytometry assay. (C) The levels of caspase-3, caspase-9, BAX, and BCL2 were determined by qRT-PCR. (D) The levels of caspase-3, cleaved caspase-3, caspase-9, cleaved caspase-9, BAX, and BCL2 were determined by Western blot assay. (E) The levels of WT1 was determined by qRT-PCR and Western blot after treating with different concentrations of NE. $(F)$ The levels of WT1 was determined by qRT-PCR and Western blot after treating with $10 \mu \mathrm{mol} / \mathrm{L} \mathrm{NE}$ for $0,6,12,24$, and $48 \mathrm{~h}$, respectively. $* P<0.05$, $* * P<0.01$ vs. control group. Data is shown as mean $\pm \mathrm{SD}(n=3)$. 
A
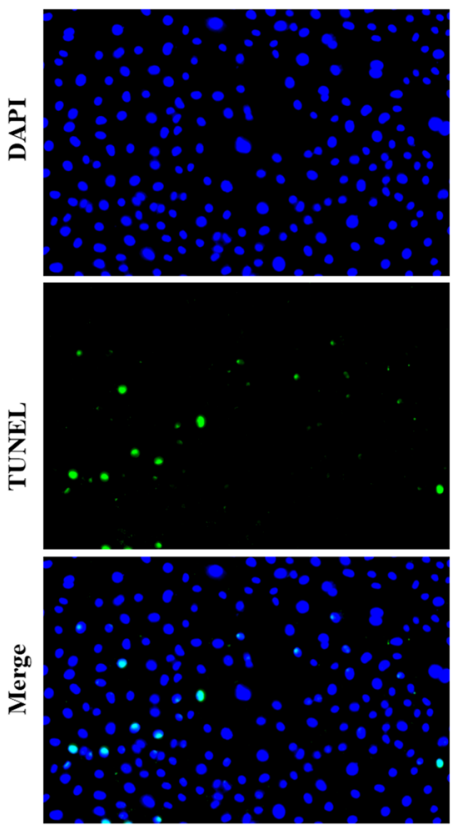

B

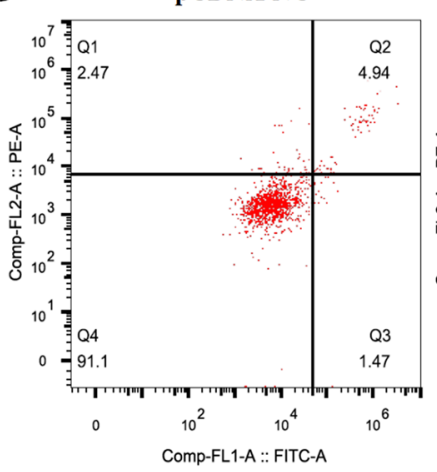

C

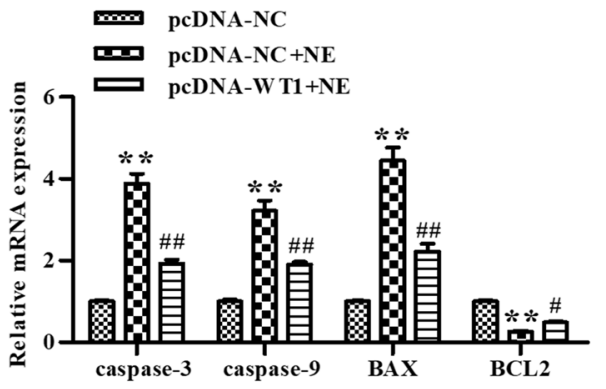

PCDNA-NC+NE
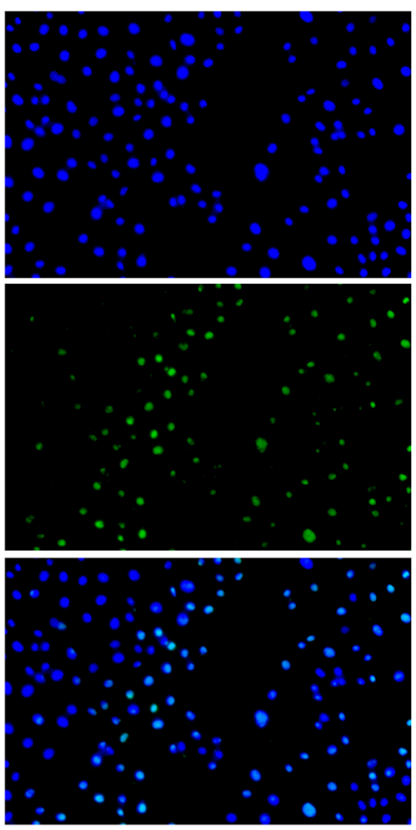

PCDNA-NC+NE

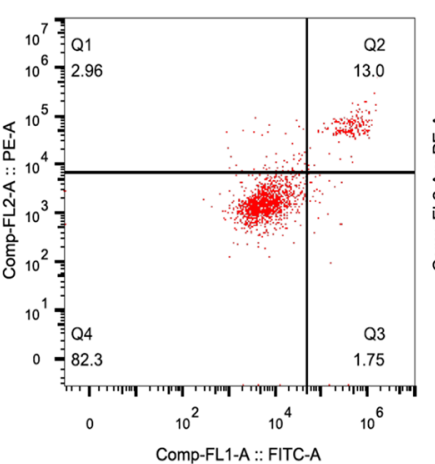

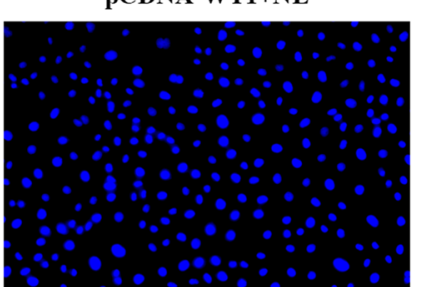
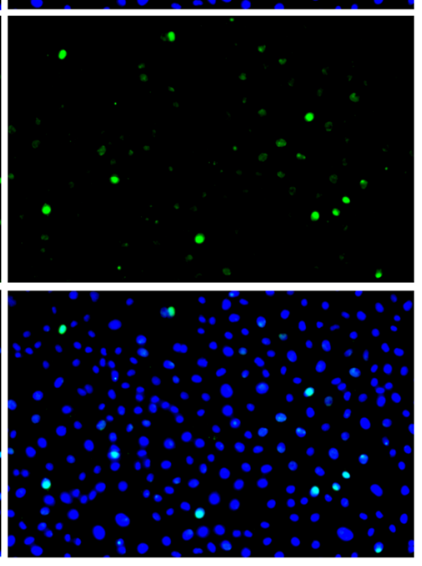

PCDNA-WT1+NE

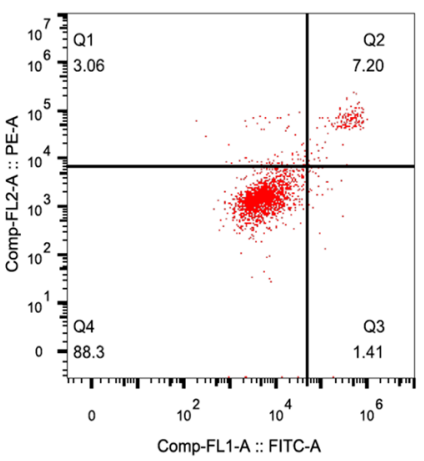

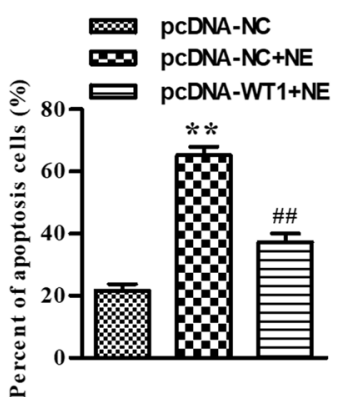

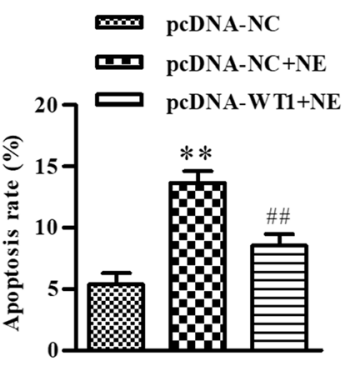

D
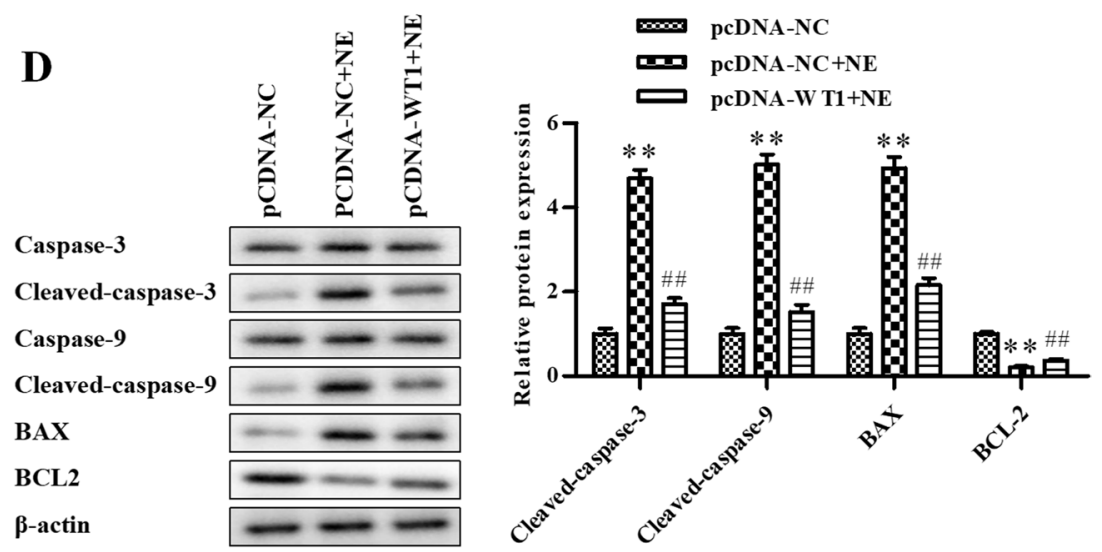

Figure 2. Overexpression of WT1 represses apoptosis in NE-treated rat GCs. (A) The cell apoptosis was determined using TUNEL assay in rat GCs. (B) The cell apoptosis was determined using flow cytometry assay. $(C)$ The levels of caspase-3, caspase-9, BAX, and BCL2 were determined by qRT-PCR. (D) The levels of caspase-3, cleaved caspase-3, caspase-9, cleaved caspase-9, BAX, and BCL2 were determined by Western blot assay. $* P<0.05, * * P<0.01$ vs. pcDNA-NC. Data is shown as mean $\pm \mathrm{SD}(n=3)$. 

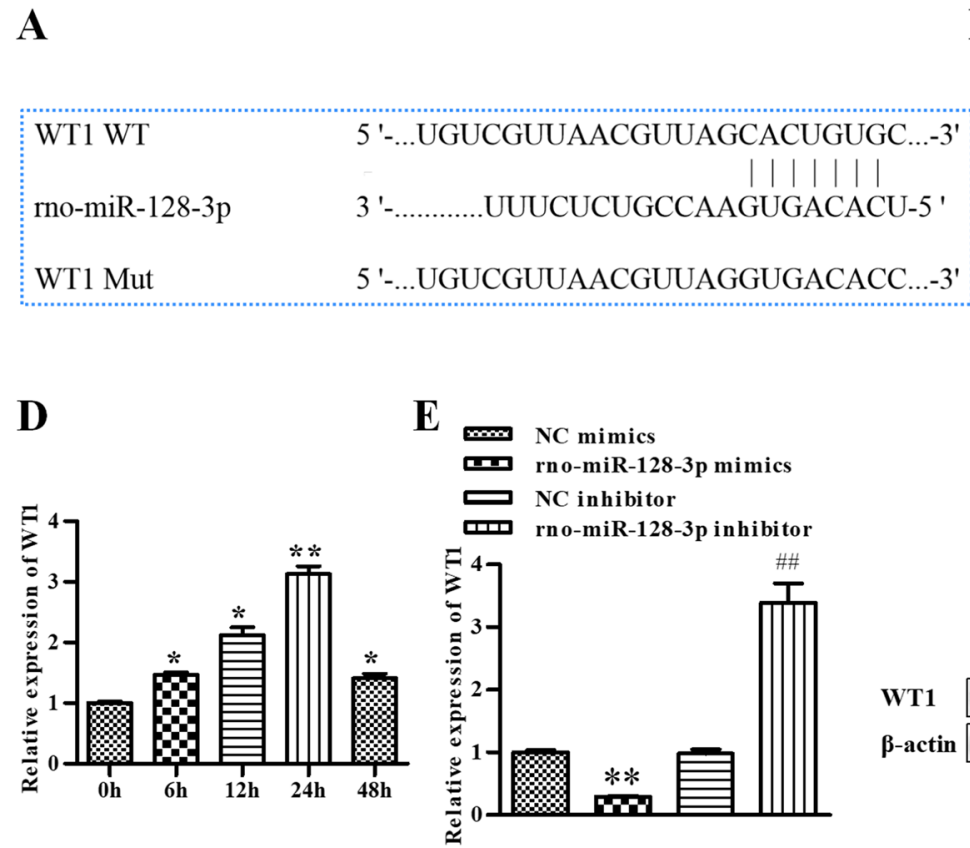

Figure 3. WT1 is a target gene of rno-miR-128-3p. (A) WT1 was predicted as a target of rno-miR-128-3p via bioinformatics analysis. (B) rno-miR-128-3p was bound to 3'UTR of WT1 using dual luciferase reporter assay, $* P<0.05, * * P<0.01$ vs. NC mimics. $(C)$ The levels of rno-miR-128-3p was determined by qRT-PCR after treating with different concentrations of NE, $* P<0.05$, $* * P<0.01$ vs. control.

WT1 is a target gene of rno-miR-128-3p WT1 was predicted as a target of rno-miR-128-3p via bioinformatics analysis (Fig. 3A), which was further verified by dual luciferase reporter assay. We co-transfected rno-miR-128-3p mimics and WT1-WT or WT1-Mut into GCs, and the data revealed that rno-miR-128-3p mimics markedly suppressed the luciferase activity of WT1-WT (Fig. 3B). Treated with different concentrations of NE, RT-qPCR was conducted to assess the level of rno-miR-128-3p. Our data exhibited that the level of rno-miR-128-3p was enhanced by NE and showed $\mathrm{NE}$ concentration dependence (Fig. 3C); then, $10 \mu \mathrm{mol} / \mathrm{L}$ $\mathrm{NE}$ was selected for subsequent experiments. Furthermore, GCs were treated with $10 \mu \mathrm{mol} / \mathrm{L}$ NE for $0,6,12,24$, and $48 \mathrm{~h}$, respectively. The results of RT-qPCR assay exhibited that the level of rno-miR-128-3p was highest for the time of $24 \mathrm{~h}$. As a result, 24-h treatment was selected for subsequent experiments (Fig. 3D).

To further verify the effect of rno-miR-128-3p on the level of WT1, we transfected GCs with rno-miR-128-3p mimics and rno-miR-128-3p inhibitor; RT-qPCR and Western blot assay were conducted to assess the level of WT1. Our data exhibited that both mRNA and protein of WT1 were repressed by rno-miR-128-3p mimics and elevated by rno-miR-128-3p inhibitor (Fig. 3E). Taken together, these
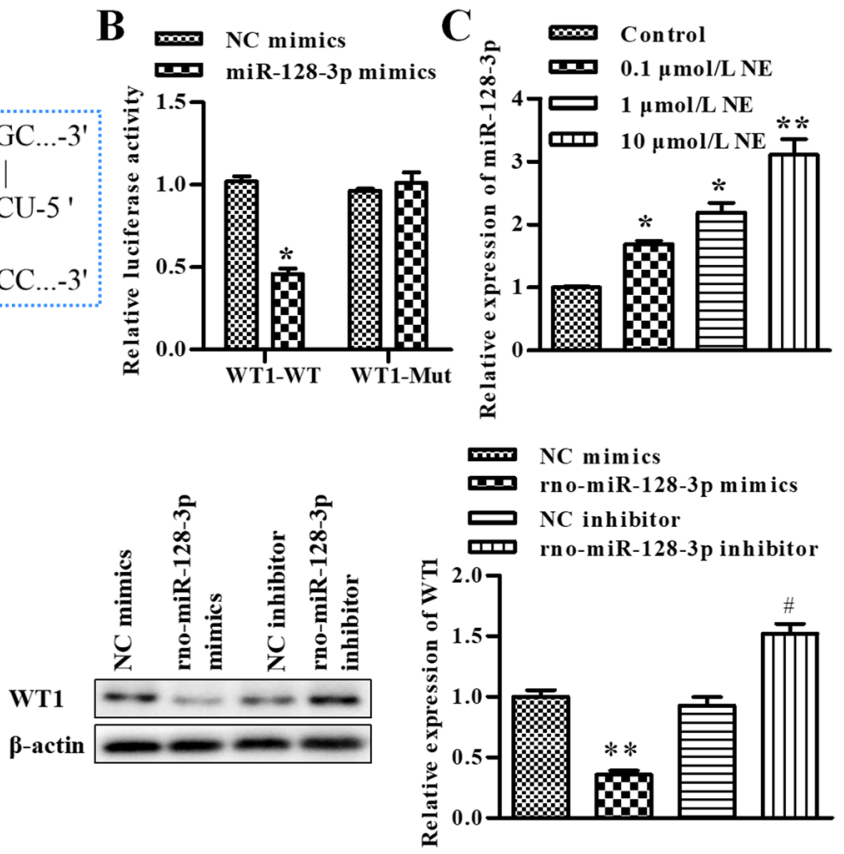

(D) The levels of rno-miR-128-3p was determined by qRT-PCR after treating with $10 \mu \mathrm{mol} / \mathrm{L} \mathrm{NE}$ for $0,6,12,24$, and $48 \mathrm{~h}$, respectively, $* P<0.05,{ }^{*} P<0.01$ vs. $0 \mathrm{~h}$. $(E)$ The level of WT1 was determined by qRT-PCR and Western blot, ${ }^{*} P<0.05, * * P<0.01$ vs. NC mimics or NC inhibitor. Data is shown as mean $\pm \mathrm{SD}(n=3)$.

results supported the idea that WT1 was a putative target of rno-miR-128-3p.

Inhibition of rno-miR-128-3p represses apoptosis in rat GCs treated with NE To further verify the effect of rno-miR128-3p on GC apoptosis, NE-treated GCs were transfected with rno-miR-128-3p inhibitor, and the TUNEL assay and flow cytometry assay were conducted to assess the apoptosis of GCs. The data revealed that NE elevated GC apoptosis compared with the NC inhibitor group, which was alleviated by rno-miR-128-3p inhibitor (Fig. $4 A$ and $B$ ). RT-qPCR was conducted to assess the levels of caspase-3, caspase-9, BAX, and BCL2, and our data exhibited that the mRNA of caspase-3, caspase-9, and BAX was significantly improved after being treated with NE, whereas it was reversed by rnomiR-128-3p inhibitor, while the results of BCL2 were the opposite (Fig. 4C). The data of the Western blot assay exhibited that the protein of cleaved caspase-3, cleaved caspase- 9 , and BAX were significantly improved by NE, whereas it was reversed by rno-miR-128-3p inhibitor and the results of BCL2 were the opposite (Fig. 4D). Taken together, GC apoptosis induced by NE was repressed by rno-miR-128-3p inhibitor.

WT1 mediates the effects of rno-miR-128-3p on NE-treated rat GCs In order to evaluate the functions of rno-miR-128-3p 

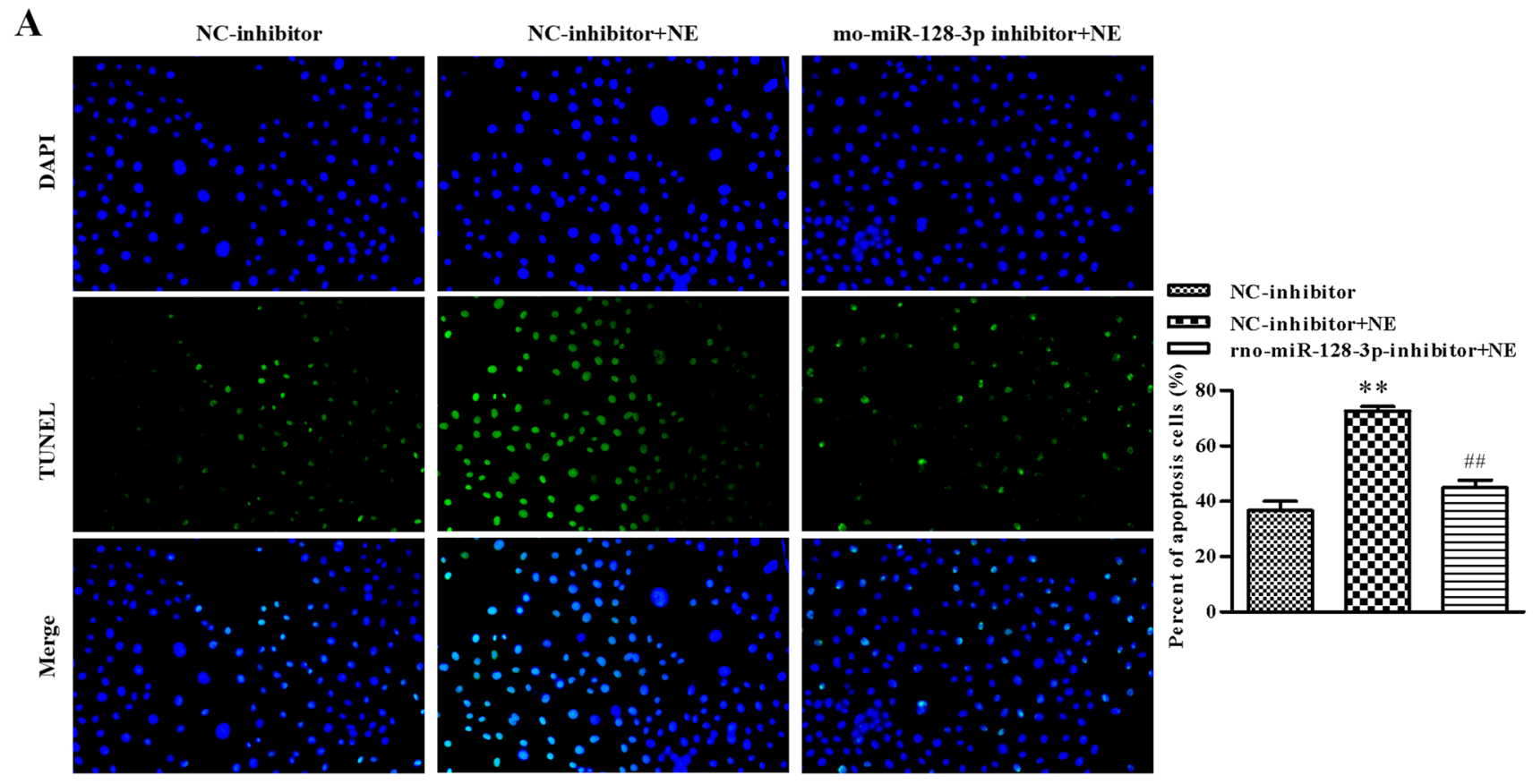
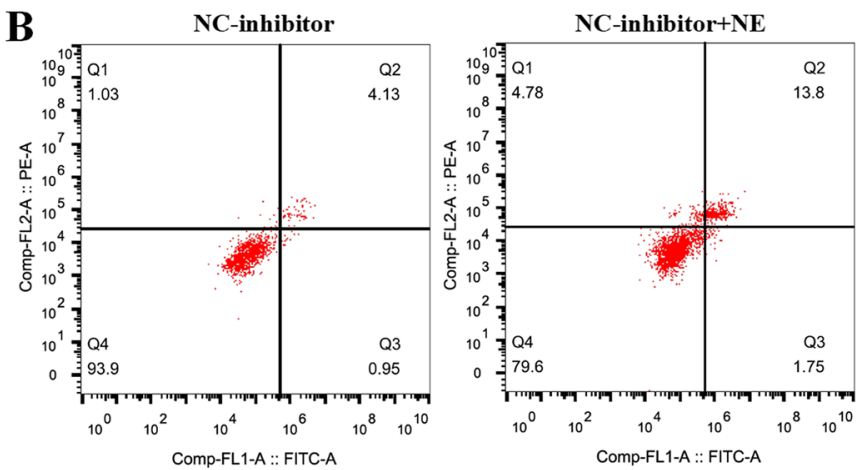

C

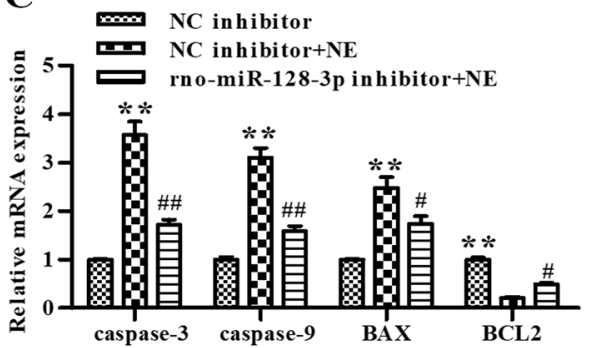

D
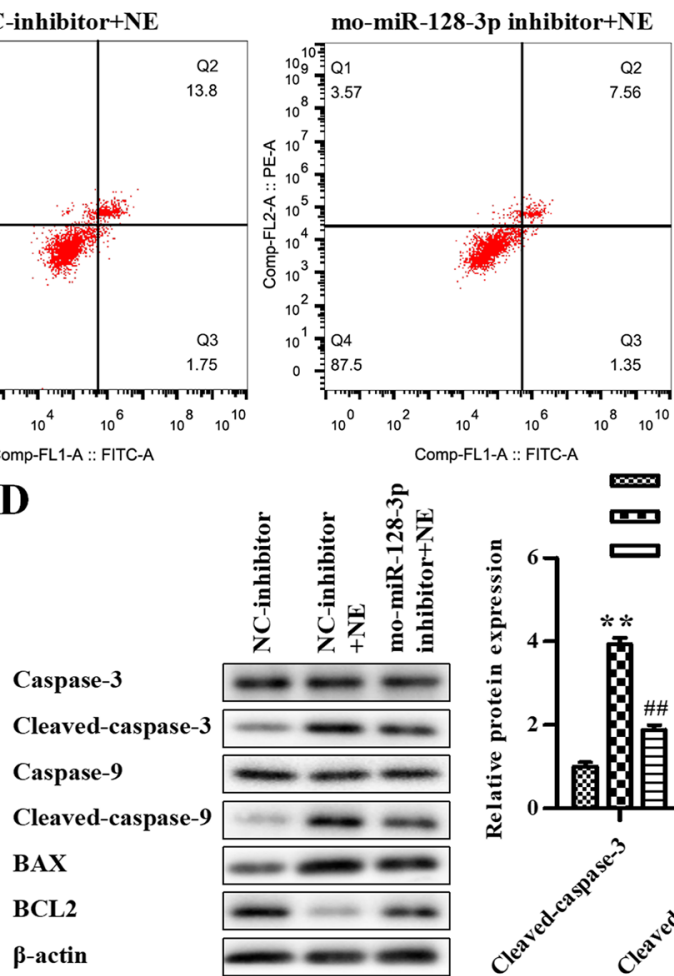

NC-inhibitor

므 NC-inhibitor+NE

rno-miR-128-3p inhibitor+NE

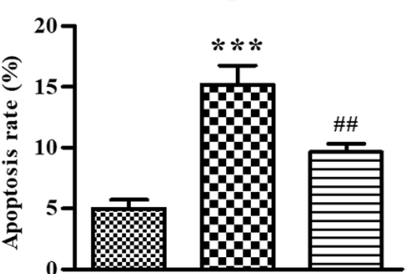

$\infty 0000$ NC inhibitor

므 NC inhibitor+NE

rno-miR-128-3p inhibitor+NE
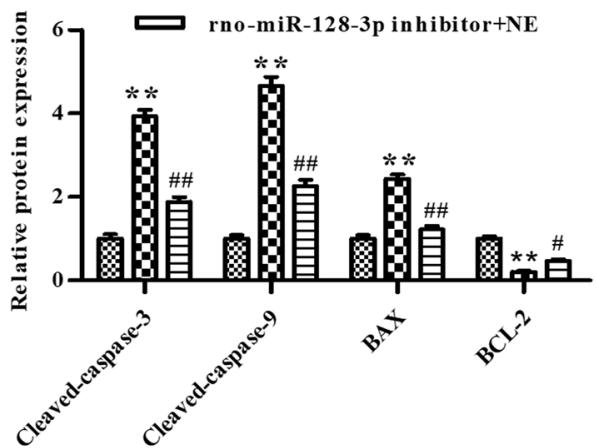

Figure 4. Inhibition of rno-miR-128-3p represses apoptosis in rat GCs treated with NE. (A) The cell apoptosis was determined using TUNEL assay in rat GCs. (B) The cell apoptosis was determined using flow cytometry assay. (C) The levels of caspase-3, caspase-9,

and WT1 in GC apoptosis induced by NE, we transfected rno-miR-128-3p inhibitor and si-WT1 into NE-treated GCs at the same time, followed by the Western blot assay. The data of Western blot assay exhibited that the protein
BAX, and BCL2 were determined by qRT-PCR. (D) The levels of caspase-3, cleaved caspase-3, caspase-9, cleaved caspase- 9 , BAX, and BCL2 were determined by Western blot assay. $* P<0.05$, $* * P<0.01$ vs. NC inhibitor. Data is shown as mean $\pm \mathrm{SD}(n=3)$.

of WT1 was observably stimulated by rno-miR-128-3p inhibitor, which was attenuated by si-WT1 (Fig. 5A). Next, the apoptosis of GCs was assessed by the TUNEL assay and flow cytometry assay. As shown in Fig. $5 B$ and $C$, the 
A

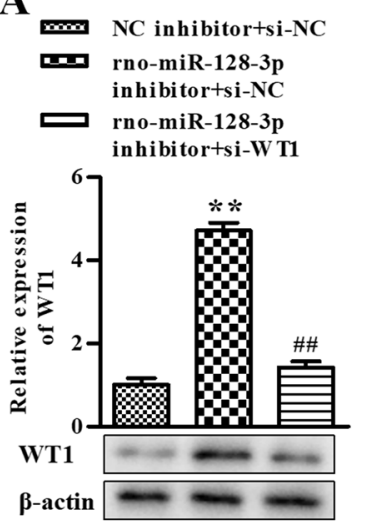

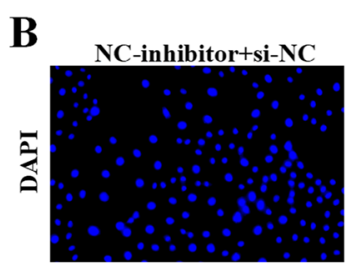
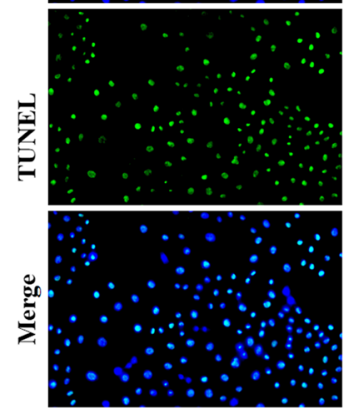

rno-miR-128-3p-
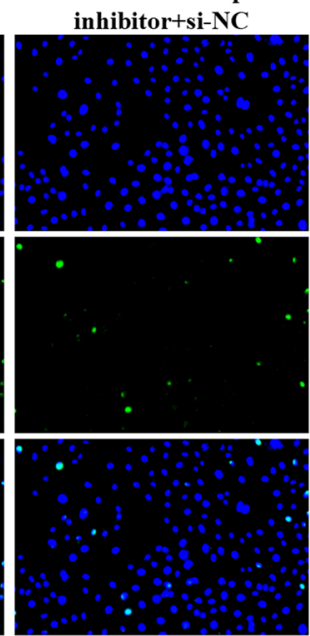
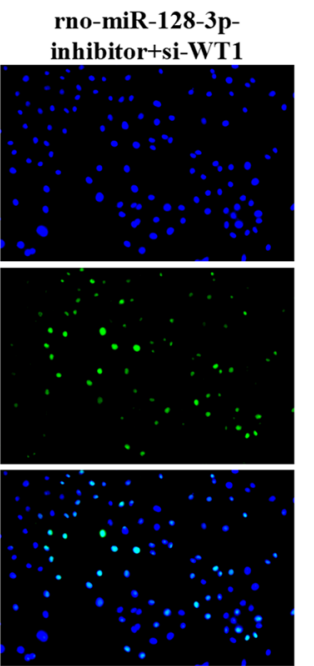

rno-miR-128-3p-

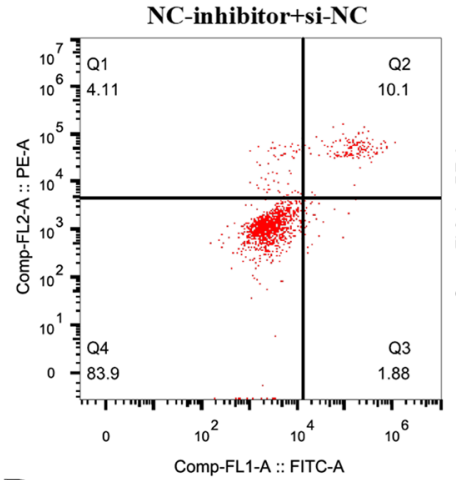

D

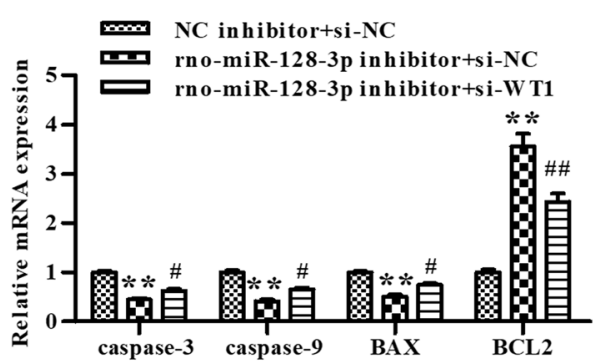

Caspase-3

Cleaved-caspase- 3 Caspase-9

Cleaved-caspase-9 BAX

BCL 2

$\beta$-actin
inhibitor+si-NC

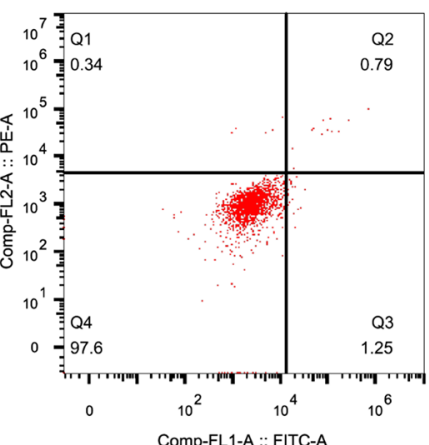

inhibitor+si-WT1

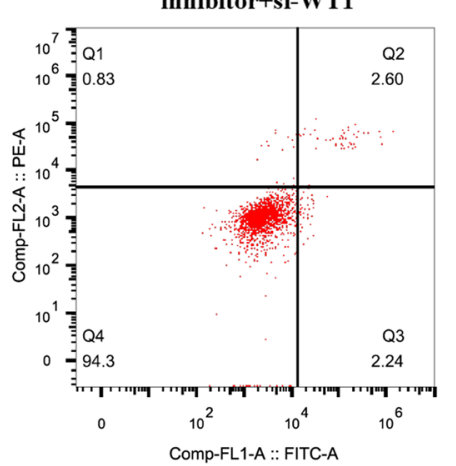

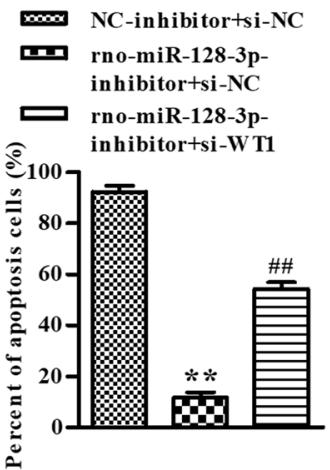

$\infty 0000$ NC-inhibitor+si-NC

드 rno-miR-128-3p inhibitor+si-NC

rno-miR-128-3p

157 inhibitor+si-WT1

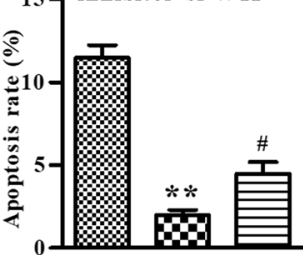

Figure 5. Inhibition of rno-miR-128-3p represses NE-induced apoptosis in rat GCs by WT1. (A) The level of WT1 was determined by Western blot. $(B)$ The cell apoptosis was determined using TUNEL assay in rat GCs. $(C)$ The cell apoptosis was determined using flow cytometry assay. (D) The levels of caspase-3, caspase-9, BAX, and
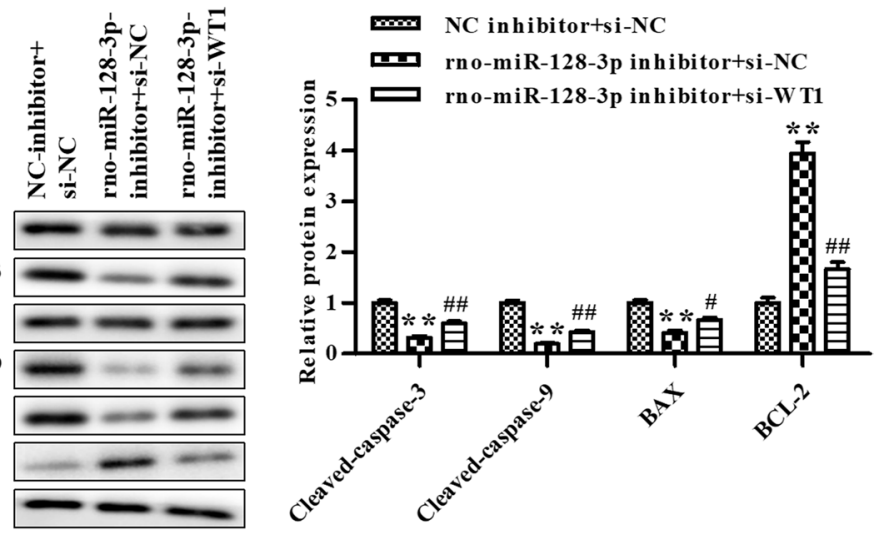

apoptosis was significantly reduced by rno-miR-128-3p inhibitor, whereas it was reversed by si-WT1. RT-qPCR was conducted to assess the levels of caspase-3, caspase-9, BAX, and BCL2, exhibiting that the mRNA of caspase-3, caspase-9, and BAX was significantly diminished by rnomiR-128-3p inhibitor, whereas it was reversed by si-WT1
BCL2 were determined by qRT-PCR. (E) The levels of caspase-3, cleaved caspase-3, caspase-9, cleaved caspase-9, BAX, and BCL2 were determined by Western blot assay. $* P<0.05$, $* * P<0.01$ vs. NC inhibitor + si-NC. Data is shown as mean $\pm \mathrm{SD}(n=3)$.

and the results of BCL2 were the opposite (Fig. 5D). The data of the Western blot assay exhibited that the protein of cleaved caspase-3, cleaved caspase-9, and BAX was significantly reduced by rno-miR-128-3p inhibitor, whereas it was reversed by si-WT1 and the results of BCL2 were the opposite (Fig. 5E). Taken together, inhibition of rno-miR-128-3p 
repressed NE-induced apoptosis in rat GCs by elevating WT1.

\section{Discussion}

Studies have proved that NE is related to various aspects of ovarian function, like early follicle development (Mayerhofer 1997), its association with the steroidogenesis, and apoptosis on dioestrus II of the ovary in rats (Bronzi et al. 2015). Recent studies indicate that NE serves as beta- 2 epinephrine receptors in rat GCs and promotes progesterone level, but it does not promote the level of estradiol (Lara 2002; Skarzynski and Kotwica 1993). NE also promotes follicle development (Mayerhofer 1997; Doganay 2010). Furthermore, it is revealed that NE represses VSMC proliferation through repressing ALK5 expression (Hu 2020). $\mathrm{NE}$ also diminishes dopaminergic cell apoptosis induced by 6-hydroxydopamine (Zhu 2019). A study conducted by Wan et al. (2018) revealed that Jujuboside A diminishes cardiomyocyte apoptosis induced by NE through the MAPK/ AKT pathway. In this study, our data demonstrated that NE induced apoptosis in rat GCs, whereas the mechanism between NE and GC apoptosis was unclear.

From this study, we found that the level of WT1 decreased in GCs after NE treatment. And apoptosis in rat GCs induced by NE was diminished after overexpression of WT1. Our result was consistent with the study conducted by Park et al. (2014), which found that WT1 repressed GC apoptosis by decreasing BAX expression. WT1 level was elevated in GCs of PCOS patient (Wang 2018); it also found that WT1 played an important role in follicle development through regulating GC differentiation and the levels of E-cadherin and Par6b (Gao 2014). WT1 is needed for lineage maintenance of GCs, and inactivation of WT leads to the transformation of preGCs into steroidogenic cells, which leads to ovarian development defects (Cen 2020).

To further verify the regulatory mechanism of WT1 in GCs, we identified that WT1 was a putative target of miR128-3p by dual luciferase reporter assay. miRNAs are a short, non-coding RNA which affect expression of target by inhibiting translation or degrading messenger RNA (Cheng 2019). Growing evidences revealed that miRNAs play a crucial role in the function of GCs. For example, miR-431 affects GC function via IRS2/PI3K/AKT pathway (Yang 2020). miR-21-3p suppresses GC autophagy of bovine through VEGFA/PI3K/AKT signaling (Ma 2019). In addition, a study conducted by Li et al. (2020) reveals that miR$146 \mathrm{~b}$ enhanced GC apoptosis by repressing CYP19A1. And another study conducted by Zhong et al. (2020) revealed that miR-204-5p improves apoptosis through attenuating Bcl2 in rat ovarian granulosa. After trans-activating by steroidogenic factor 1, miR-202-5p promotes apoptosis of goat GCs through regulating TGF $\beta$ R2 (Ding et al. 2020). And it finds that miR-30d-5p promotes GC apoptosis through suppressing Smad2 (Yu and Liu 2020). Likewise, our data demonstrated that inhibition of miR-128-3p repressed apoptosis in rat GCs induced by NE.

In summary, our finding elucidated that rno-miR-128-3p was enhanced and WT1 was alleviated in NE-treated GCs. Inhibition of rno-miR-128-3p repressed apoptosis in rat GCs induced by NE. Therefore, the miR-128-3p/WT1 pathway may serve as a potential mechanism for NE-induced GC apoptosis, and our finding may provide a new insight for the improvement of ovarian follicular development.

Funding This work was supported by Jiangsu Pharmaceutical Association-Tianqing Hospital Pharmaceutical Fund [Q202046], and Health Science and Technology Project of Lianyungang City [ZD1906].

Data availability All data generated or analyzed during this study are included in this published article.

\section{Declarations}

Consent for publication The authors consent for publication in the Journal.

Competing interests The authors declare no competing interests.

Open Access This article is licensed under a Creative Commons Attribution 4.0 International License, which permits use, sharing, adaptation, distribution and reproduction in any medium or format, as long as you give appropriate credit to the original author(s) and the source, provide a link to the Creative Commons licence, and indicate if changes were made. The images or other third party material in this article are included in the article's Creative Commons licence, unless indicated otherwise in a credit line to the material. If material is not included in the article's Creative Commons licence and your intended use is not permitted by statutory regulation or exceeds the permitted use, you will need to obtain permission directly from the copyright holder. To view a copy of this licence, visit http://creativecommons.org/licenses/by/4.0/.

\section{References}

Bronzi CD, Orozco ASV, Rodriguez D, Rastrilla AM, Sosa ZY, Casais, (2015) Noradrenaline modulates the presence of gonadotropinreleasing hormone in ovary. The importance of its interrelation on the ovarian steroidogenesis and apoptosis on dioestrus II in rat. J Steroid Biochem Mol Biol 154:39-46

Cen C, Chen M, Zhou J, Zhang L, Duo S, Jiang L, Hou X, Gao F (2020) Inactivation of Wt1 causes pre-granulosa cell to steroidogenic cell transformation and defect of ovary development $\dagger$. Biol Reprod 103(1):60-69

Chen M, Zhnag L, Cui X, Lin X, Li Y, Wang Y, Wang Y, Qin Y, Chen D, Han C, Zhou B, Huff V, Gao F (2017) Wt1 directs the lineage specification of sertoli and granulosa cells by repressing $\mathrm{Sf} 1$ expression. Development (cambridge, England) 144(1):44-53

Cheng Y, Qiu L, He G-L, Cai L, Peng B-J, Cao Y-L, Pan M-X (2019) MicroRNA-361-5p suppresses the tumorigenesis of hepatocellular carcinoma through targeting WT1 and 
suppressing WNT/ $\beta$-cadherin pathway. Eur Rev Med Pharmacol Sci 23(20):8823-8832

Choi MJ, Cho KH, Lee S, Bae YJ, Jeong KJ, Rha SY, Choi EJ, Park JH, Kim JM, Lee J-S, Mills GB, Lee HY (2015) hTERT mediates norepinephrine-induced Slug expression and ovarian cancer aggressiveness. Oncogene 34(26):3402-3412

Chowdhury I, Thompson WE, Welch C, Thomas K, Matthews R (2013) Prohibitin (PHB) inhibits apoptosis in rat granulosa cells (GCs) through the extracellular signal-regulated kinase 1/2 (ERK1/2) and the Bcl family of proteins. Apoptosis : an International Journal on Programmed Cell Death 18(12):1513-1525

Chun SY, McGee EA, Hsu SY, Minami S, LaPolt PS, Yao HH, Bahr JM, Gougeon A, Schomberg DW, Hsueh AJ (1999) Restricted expression of WT1 messenger ribonucleic acid in immature ovarian follicles: uniformity in mammalian and avian species and maintenance during reproductive senescence. Biol Reprod 60(2):365-373

del Campo M, Piquer B, Witherington J, Sridhar A, Lara HE (2019) Effect of superior ovarian nerve and plexus nerve sympathetic denervation on ovarian-derived infertility provoked by estradiol exposure to rats. Front Physiol 10:349

Ding Q, Jin M, Wang Y, Liu J, Kalds P, Wang Y, Yang Y, Wang X, Chen Y (2020) Transactivation of miR-202-5p by steroidogenic factor 1 (SF1) induces apoptosis in goat granulosa cells by targeting TGFßR2. Cells 9(2)

Doganay M, Simsek A, Tapisiz OL, Mulazimoglu BS, Yumusak N, Gungor T (2010) Superior ovarian nerve (SON) transection leads to stunted follicular maturation: a histomorphologic and morphometric analysis in the rat model. Fertil Steril 93(5):1711-1714

Feng S, Sun H, Zhu W (2020) MiR-92 overexpression suppresses immune cell function in ovarian cancer via LATS2/YAP1/PD-L1 pathway. Clinical \& translational oncology : official publication of the Federation of Spanish Oncology Societies and of the National Cancer Institute of Mexico

Fortune JE (1994) Ovarian follicular growth and development in mammals. Biol Reprod 50(2):225-232

Gao F, Zhang J, Wang X, Yang J, Chen D, Huff V, Liu Y-X (2014) Wt1 functions in ovarian follicle development by regulating granulosa cell differentiation. Hum Mol Genet 23(2):333-341

Harishkumar R, Selvaraj CI (2020) Lotusine, an alkaloid from Nelumbo nucifera (Gaertn.), attenuates doxorubicin-induced toxicity in embryonically derived H9c2 cells. In vitro Cell Dev Biol Animal 56(5):367-377

Hu X, Miao J, Zhang M, Wang X, Wang Z, Han J, Tong D, Huang C (2018) In vitro miRNA-103a-3p promotes human gastric cancer cell proliferation by targeting and suppressing ATF7. Mol Cells 41(5):390-400

Hu Z, Li B, Wang Z, Hu X, Zhang M, Chen R, Wu Q, Jia F (2020) The sympathetic transmitter norepinephrine inhibits VSMC proliferation induced by TGF $\beta$ by suppressing the expression of the TGF $\beta$ receptor ALK5 in aorta remodeling. Mol Med Rep 22(1):387-397

Huang X, Wu B, Chen M, Hong L, Kong P, Wei Z, Teng X (2020) Depletion of exosomal circLDLR in follicle fluid derepresses miR1294 function and inhibits estradiol production via CYP19A1 in polycystic ovary syndrome. Aging. 12

Jiang L, Huang H, Qian Y, Li Y, Chen X, Di N, Yang D (2020) miR$130 \mathrm{~b}$ regulates gap junctional intercellular communication through connexin 43 in granulosa cells from patients with polycystic ovary syndrome. Molecular human reproduction

Lara HE, Dorfman M, Venegas M, Luza SM, Luna SL, Mayerhofer A, Guimaraes MA, Silva Rosa E, AAM, Ramírez VD, (2002) Changes in sympathetic nerve activity of the mammalian ovary during a normal estrous cycle and in polycystic ovary syndrome: Studies on norepinephrine release. Microsc Res Tech 59(6):495-502
Li Q, Du X, Liu L, Liu H, Pan Z, Li Q (2020) Upregulation of miR$146 \mathrm{~b}$ promotes porcine ovarian granulosa cell apoptosis by attenuating CYP19A1. Domest Anim Endocrinol 74:106509

Li G-Q, Fang Y-X, Liu Y, Meng F-R, Wu X, Zhang C-H, Zhang Y, Liu D, Gao B (2019b) MALAT1-driven inhibition of Wnt signal impedes proliferation and inflammation in fibroblast-like synoviocytes through CTNNB1 promoter methylation in rheumatoid arthritis. Hum Gene Ther 30(8):1008-1022

Li J, Chen Y, Shen L, Deng Y (2019c) Improvement of membranous nephropathy by inhibition of miR-193a to affect podocytosis via targeting WT1. J Cell Biochem 120(3):3438-3446

Li T-S, Chen L, Wang S-H, Yang Y-Z, Xu H-J, Gu H-M, Zhao X-J, Dong P, Pan Y, Shang Z-Q, Zhang X-Q, Kong L-D (2019a) Magnesium isoglycyrrhizinate ameliorates fructose-induced podocyte apoptosis through downregulation of miR-193a to increase WT1. Biochem Pharmacol 166:139-152

Ma L, Zheng Y, Tang X, Gao H, Liu N, Gao Y, Hao L, Liu S, Jiang Z (2019) miR-21-3p inhibits autophagy of bovine granulosa cells by targeting VEGFA via PI3K/AKT signaling. Reproduction (cambridge, England) 158(5):441-452

Mayerhofer A, Dissen GA, Costa ME, Ojeda SR (1997) A role for neurotransmitters in early follicular development: induction of functional follicle-stimulating hormone receptors in newly formed follicles of the rat ovary. Endocrinology 138(8):3320-3329

Murray A, Spears N (2000) Follicular development in vitro. Semin Reprod Med 18(2):109-122

Park M, Choi Y, Choi H, Roh J (2014) Wilms' tumor suppressor gene (WT1) suppresses apoptosis by transcriptionally downregulating BAX expression in immature rat granulosa cells. J Ovar Res 7:118

Patel PR, Hegde ML, Theruvathu J, Mitra SA, Boldogh I, Sowers L (2015) Norepinephrine reduces reactive oxygen species (ROS) and DNA damage in ovarian surface epithelial cells. J Bioanal Biomed 7(3):75-80

Pelletier J, Schalling M, Buckler AJ, Rogers A, Haber DA, Housman D (1991) Expression of the Wilms' tumor gene WT1 in the murine urogenital system. Genes Dev 5(8):1345-1356

Saller S, Merz-Lange J, Raffael S, Hecht S, Pavlik R, Thaler C, Berg D, Berg U, Kunz L, Mayerhofer A (2012) Norepinephrine, active norepinephrine transporter, and norepinephrine-metabolism are involved in the generation of reactive oxygen species in human ovarian granulosa cells. Endocrinology 153(3):1472-1483

Shen H, Wang Y (2019) Activation of TGF- $\beta 1 /$ Smad3 signaling pathway inhibits the development of ovarian follicle in polycystic ovary syndrome by promoting apoptosis of granulosa cells. J Cell Physiol 234(7):11976-11985

Skarzynski D, Kotwica J (1993) Mechanism of noradrenaline influence on the secretion of ovarian oxytocin and progesterone in conscious cattle. J Reprod Fertil 97(2):419-424

Van Voorhis BJ (2008) Ultrasound assessment of the ovary in the infertile woman. Semin Reprod Med 26(3):217-222

Wan C-H, Han D-D, Xu J-Q, Yin P, Xu X-L, Mei C, Liu F-H, Xia Z-F (2018) Jujuboside A attenuates norepinephrine-induced apoptosis of $\mathrm{H} 9 \mathrm{c} 2$ cardiomyocytes by modulating MAPK and AKT signaling pathways. Mol Med Rep 17(1):1132-1140

Wang J, Qiu J, Bo Le, Wu Z, Zhou A, Xu W, Mao C (2019) WT1 influences apoptosis and proliferation of immature mice granular cells through regulation of the wnt/ $\beta$-catenin signal pathway. Cellular and molecular biology (Noisy-le-Grand, France) 65(7):138-145

Wang J, Wu Q, Yu J, Cao X, Xu Z (2019) miR-125a-5p inhibits the expression of NLRP3 by targeting CCL4 in human vascular smooth muscle cells treated with ox-LDL. Exp Ther Med 18(3):1645-1652

Wang P, Liu S, Zhu C, Duan Q, Jiang Y, Gao K, Bu Q, Cao B, An X (2020) MiR-29 regulates the function of goat granulosa cell by targeting PTX3 via the PI3K/AKT/mTOR and Erk1/2 signaling 
pathways. The Journal of steroid biochemistry and molecular biology 202:105722

Wang Q, Huang T, Shu X, Zhao S-G, Liang Y, Muhammad T, Gao F, Zhao H, Liu H-B (2018) Wilms' tumor 1 overexpression in granulosa cells is associated with polycystic ovaries in polycystic ovary syndrome patients. Gynecol Obstet Invest 83(3):241-246

Webb R, Nicholas B, Gong JG, Campbell BK, Gutierrez CG, Garverick HA, Armstrong DG (2003) Mechanisms regulating follicular development and selection of the dominant follicle. Reprod Suppl 61:71-90

Woo I, Christenson LK, Gunewardena S, Ingles SA, Thomas S, Ahmady A, Chung K, Bendikson K, Paulson R, McGinnis LK (2018) Micro-RNAs involved in cellular proliferation have altered expression profiles in granulosa of young women with diminished ovarian reserve. J Assist Reprod Genet 35(10):1777-1786

Wu M-B, Ma B, Zhang T-X, Zhao K, Cui S-M, He S-C (2020) Propofol improves intestinal ischemia-reperfusion injury in rats through NF-кB pathway. Eur Rev Med Pharmacol Sci 24(11):6463-6469

Yang L, Lv Q, Liu J, Qi S, Fu D (2020) miR-431 regulates granulosa cell function through the IRS2/PI3K/AKT signaling pathway. J Reprod Dev 66(3):231-239

Yu M, Liu J (2020) MicroRNA-30d-5p promotes ovarian granulosa cell apoptosis by targeting Smad2. Exp Ther Med 19(1):53-60
Zhang X, Zhang Y, Cai W, Liu Y, Liu H, Zhang Z, Su Z (2020) MicroRNA-128-3p alleviates neuropathic pain through targeting ZEB1. Neuroscience letters 729: 134946

Zhang L, Gao J, Cui S (2017a) miR-21 is involved in norepinephrinemediated rat granulosa cell apoptosis by targeting SMAD7. J Mol Endocrinol 58(4):199-210

Zhang L, Gao J, Cui S (2017b) SMAD7miR-21 is involved in norepinephrine-mediated rat granulosa cell apoptosis by targeting. J Mol Endocrinol 58(4):199-210

Zhang X, Gan X, Qiannan E, Zhang Q, Ye Y, Cai Y, Han A, Tian M, Wang Y, Wang C, Su L, Liang C (2019) Ameliorative effects of nano-selenium against NiSO-induced apoptosis in rat testes. Toxicol Mech Methods 29(7):467-477

Zhong P, Liu J, Li H, Lin S, Zeng L, Luo L, Wu M, Zhang W (2020) MicroRNA-204-5p regulates apoptosis by targeting $\mathrm{Bcl} 2$ in rat ovarian granulos $a$. Biol Reprod

Zhu M-Y, Raza MU, Zhan Y, Fan Y (2019) Norepinephrine upregulates the expression of tyrosine hydroxylase and protects dopaminegic neurons against 6-hydrodopamine toxicity. Neurochem Int 131:104549 